\title{
Association of One-Leg Standing Time with Discontinuation of Injectable Medications During Hospitalization Among Patients with Type 2 Diabetes
}

\author{
Kazuhiro Sugimoto $\cdot$ Yuji Tanaka - Takashi Sozu · Hiroshi Nishiyama • \\ Takehiko Hoshino Yuko Watanabe · Akira Tamura - Setsu Ohta • \\ Toshiro Yamazaki · Susumu Suzuki · Takuro Shimbo
}

Received: January 13, 2020 / Published online: April 9, 2020

(C) The Author(s) 2020

\section{ABSTRACT}

Introduction: Persons with type 2 diabetes (T2D) are known to experience impaired physical ability even at the early stages of the disease. However, less attention has been paid to increasing physical ability than to increasing physical activity in the treatment of T2D. The aim of this study was to assess whether

Digital features To view digital features for this article go to https://doi.org/10.6084/m9.figshare.12040566.

K. Sugimoto $(\bowtie) \cdot$ Y. Watanabe · A. Tamura .

S. Ohta $\cdot$ T. Yamazaki $\cdot$ S. Suzuki

Diabetes Center, Ohta Nishinouchi Hospital,

Koriyama, Japan

e-mail: sugimoto@ohta-hp.or.jp

Y. Tanaka

Department of Management Science, Tokyo

University of Science Graduate School of

Engineering, Tokyo, Japan

\section{T. Sozu}

Department of Information and Computer

Technology, Faculty of Engineering, Tokyo

University of Science, Tokyo, Japan

H. Nishiyama

Medicines Development Unit-Japan and Medical

Affairs, Eli Lilly Japan K.K, Kobe, Japan

\section{T. Hoshino}

Department of Physical Training and Science, Ohta

Nishinouchi Hospital, Koriyama, Japan

T. Shimbo

Ohta Nishinouchi Hospital, Koriyama, Japan improved physical ability parameters are associated with the discontinuation of injectable medications once glycemic targets have been achieved among inpatients with inadequately controlled T2D across a wide range of ages.

Methods: Forty-three patients with glycated hemoglobin levels of $\geq 7.5 \%(58 \mathrm{mmol} / \mathrm{mol})$ aged between 19 and 82 years who received insulin, glucagon-like peptide-1 receptor agonists or both at admission were enrolled in the study. Muscle strength for knee extension, oneleg standing time with eyes open test(OLST), whole-body reaction time and maximal oxygen uptake were assessed as parameters of physical ability.

Results: At admission, patients who during hospitalization discontinued injectable medications ( $n=29$; Discontinued group) had a shorter duration of diabetes, lower fat mass and higher skeletal muscle mass and performed better on all of the physical ability test parameters than those who continued on injectable medications during hospitalization ( $n=14$; Continued group). At discharge, patients in the Discontinued group had achieved better glycemic control than those in the Continued group, as indicated by lower mean plasma glucose levels according to the 7-point profile. Stepwise logistic regression analysis that included those variables that were significantly different between the Continued group and the Discontinued group, with the 
aim to identify candidate(s) of explanatory variables, revealed that only OLST was significantly associated with the discontinuation of injectable medication. Patients with an OLST of $\geq 60 \mathrm{~s}$ were more likely to discontinue injectable medication than those with an OLST of $<60 \mathrm{~s}$ (odds ratio 18.9; 95\% confidence interval 2.0-178.8; $p=0.011$ ).

Conclusions: Among inpatients with inadequately controlled T2D diabetes, longer OLST appear to be associated with discontinuing injectable medications during hospitalization. OLST could possibly be useful as a novel patient factor to consider in de-intensifying injectable medication.

Keywords: Functional balance; Glucagon-like peptide-1 receptor agonist; Glycemic management; Insulin; Physical ability; Type 2 diabetes

\section{Key Summary Points}

\section{Why carry out this study?}

Less attention has been paid to increasing physical ability than to increasing physical activity in the treatment of type 2 diabetes (T2D), despite T2D patients experiencing impaired physical ability even at the early stages of the disease.

The aim of this study was to investigate whether increased physical ability is associated with the discontinuation of injectable medications once inpatients with poorly controlled T2D across a wide range of ages have achieved glycemic control.

\section{What was learned from the study?}

Patients who discontinued injectable medications performed better on all of the physical ability test parameters examined at admission and had achieved better glycemic control at discharge than those who continued injectable medications.
Stepwise logistic regression analysis including diabetes duration, total daily insulin doses and the physical ability parameters as explanatory variables revealed that only the one-leg standing time with eyes open, a simple measure of functional balance, was significantly associated with discontinuation of injectable medication in these patients.

\section{INTRODUCTION}

Much attention has been directed toward the beneficial effects of increasing physical activity and exercise in persons with type 2 diabetes (T2D) [1]. Most current guidelines have recommended that patients with T2D undertake at least 150 min per week of physical activity in order to improve glycemic control $[2,3]$. However, less attention has been paid to impaired physical ability in the treatment of T2D, despite reliable data showing that muscle function can be affected even during the early stages of the disease [4] and that lower grip strength is associated with a higher risk of diabetes [5]. In addition, although this impaired physical ability is reportedly linked to hyperglycemia in older individuals [6], little is known about whether this assocation is restricted to older patients or whether it is present in patients of all ages.

Our group has previously demonstrated that exercise training results in a significant reduction of the amount of oral hypoglycemic agents required by hospitalized patients with $\mathrm{T} 2 \mathrm{D}$ and leads to the discontinuation of insulin therapy in this patient population [7]. However, to our knowledge, no studies have investigated the relationship between physical ability and the pharmacologic therapy needed to achieve glycemic goals in these patients. This information could be useful as a novel patient factor to determine and titrate the medication regimens needed for individualized glycemic management. Injectable glucagon-like peptide-1 receptor agonists (GLP-1RAs) reportedly exhibit 
similar [8] or even better glucose-lowering effects [9] than insulin in patients with inadequately controlled T2D. Current guidelines recommend that, when possible, GLP-1RAs are preferred to insulin among patients with inadequately controlled T2D who need greater glucose lowering that can be obtained with oral medications [10]. Consequently, it may be worth exploring whether increased physical ability is associated with the discontinuation of injectable medications, including GLP-1RAs, particularly among patients with inadequately controlled T2D.

The aim of this study was to investigate whether the one-leg standing time with eyes open (OLST) test (referred to simply as OLST in subsequent text) is associated with discontinuation of the injectable medications needed to achieve glycemic goals among inpatients with inadequately controlled T2D across a wide range of ages. To safeguard patient safety, before prescribing the supervised exercise programs during the hospital stay, we performed a routine physical evaluation of the patients enrolled in the study using a battery of physical ability tests, including an OLST test, as described in detail in the Methods section.

\section{METHODS}

\section{Study Data}

All analyses were conducted with the approval of the Ethical Committee of the Ohta Nishinouchi Hospital (approval no. 3; approval date 18 June 2018) and in accordance with Helsinki Declaration of 1964 and its later amendments as well as with the ethical guidelines for medical and health research involving human subjects issued by the Ministry of Health, Labour and Welfare of Japan in 2017.

Between December 2012 and February 2020, 112 patients who had T2D but not diabetes due to any other causes, such as type 1 diabetes, diabetes secondary to chronic pancreatitis or steroid diabetes, were admitted to the wards of Ohta Nishinouchi Hospital. At admission, all patients were receiving injectable medications (i.e., insulin, GLP-1RAs or both) for management of their T2D and were being treated by a diabetologist (KS). The most common reason for hospitalization was the willingness of the patients to be admitted to hospital in an effort to improve and achieve an effective diabetes self-management ability, typically in the presence of poor glycemic control but not due to hyperglycemic crisis (diabetic ketoacidosis or hyperglycemic hyperosmolar state) or any other condition (e.g. acute infections or illnesses). Clinical data were collected on these patients, including medical history, medication use and physical ability parameters (as further in this section).

We excluded patients with T2D who were expected to require insulin therapy due to the following conditions at admission: glycosuria accompanied by ketonuria; fasting serum C-peptide levels of $<0.20 \mathrm{nmol} / \mathrm{l}$, indicating the presence of absolute insulin deficiency or undiagnosed type 1 diabetes [11]; C-reactive protein of $\geq 3 \mathrm{mg} / \mathrm{dl}$ or erythrocyte sedimentation rate of $\geq 100 \mathrm{~mm}$ after $2 \mathrm{~h}$, indicating underlying inflammatory diseases; estimated glomerular filtration rate (eGFR) of $<45 \mathrm{ml} /$ $\min / 1.73 \mathrm{~m}^{2}$, indicating the presence of impaired renal function; endocrine disorders; malignant neoplasms; or liver cirrhosis. Patients who could not participate in the exercise programs due to significant comorbidities, such as orthopedic problems, cerebrovascular diseases and/or heart failure, were also excluded from the study since they might need the greater potency of injectable medications to achieve glycemic goals. In addition, patients were also excluded if their hemoglobin A1c (HbA1c) levels were $<7.5 \%(58 \mathrm{mmol} / \mathrm{mol})$, if their plasma glucose levels were $<3.9 \mathrm{mmol} / \mathrm{l}$ or if they had newly diagnosed T2D (known duration of diabetes $<1$ year), had discontinued treatment or had ever been admitted to our hospital ward.

Of the initial 112 patients assessed, 43 patients with T2D aged between 19 and 82 years were identified as eligible to participate in the study, and available data on these 43 patients were retrieved and analyzed anonymously. During the hospital stay, glucose-lowering therapy was initiated to achieve the best possible glycemic control without hypoglycemia 
based on the consensus statements from the American Diabetes Association and the European Association for the Study of Diabetes. In addition, injectable medication was discontinued so long as the glycemic target was maintained as follows: preprandial and 2-h postprandial plasma glucose (PG) was 4.4-$7.2 \mathrm{mmol} / \mathrm{l}$ and $<10.0 \mathrm{mmol} / \mathrm{l}$, respectively.

\section{Body Composition and Physical Ability}

At the beginning of hospitalization, the body composition and physical ability of each patient were assessed. A multifrequency bioelectrical impedance analyzer (InBody 720; Biospace, Tokyo, Japan) was used to assess body composition (fat mass and skeletal muscle mass). Although bioelectrical impedance analysis has limitations in terms of inter- and intra-individual variability due to changes in the chemical composition of fat-free mass that occur under various conditions [12], this method can be used for measuring body composition as a comparable alternative to the "gold-standards," such as hydrodensitometry and dual-energy $\mathrm{X}$-ray absorptiometry [13].

Maximal isometric muscle strength for knee extension was determined using a isometric knee musculometer (Hydromusculator GT-160; OG Giken, Okayama, Japan). Strength was normalized to leg muscle mass in kilograms to give a measure of leg muscle quality [14]. Leg muscle mass was determined by using the InBody 720 impedance analyzer. OLST was recorded, up to a maximum of $60 \mathrm{~s}$, to assess functional balance. Muscle strength, muscle quality and OLST were measured for both legs. The average values for each leg were then calculated and the results used for analysis since the bilateral difference was almost zero (data not shown). The OLST was truncated at and inflated to $60 \mathrm{~s}$; as such, it was divided into dichotomous groups: 0 to $<60 \mathrm{~s}$ and $\geq 60 \mathrm{~s}$. To assess agility performance, we measured wholebody reaction time (TKK-1264b; Takei Scientific Instruments, Niigata, Japan), as previously described with minor modifications [15]. We used the shortest time from the sound stimulus to complete lifting of each foot from the floor in five consecutive trials with little or no recovery time. Maximal oxygen uptake was estimated to assess cardiopulmonary fitness and was estimated using a computerized metabolic monitor (Aeromonitor AE-300S; Minato Medical Science, Osaka, Japan) during graded exercise on a bicycle ergometer (Well Bike BE-360; Fukuda Denshi, Tokyo, Japan) at an intensity of up to $80 \%$ of the age-predicted maximal heart rate (220 beats per minute - age).

\section{Glycemic Status}

The mean PG level was calculated from the 8-point and 7-point profiles at admission and discharge, respectively. These profiles were measured at the designated times, namely, before and $2 \mathrm{~h}$ after breakfast, lunch and dinner and before bedtime (2200 hours) (for the 7 - and 8-point profiles) plus early morning (0400 hours) (only for the 8-point profiles).

\section{Statistical Analysis}

Continuous variables were summarized using the mean with the standard deviation (SD), and categorical variables were summarized using counts and proportions. Differences in clinical characteristics between patients who continued their treatment with injectable medications, including insulin, GLP-1RA, or both (Continued group), and those who discontinued treatment with injectable medications (Discontinued group) during hospitalization were compared using the $t$ test or Fisher's exact test. A stepwise logistic regression analysis included significantly different variables between the Continued group and the Discontinued group and was applied to select significant variables associated with discontinuation of the injectable medication. In this analysis, odds ratios (ORs) with 95\% confidential intervals (CIs) of injectable medication discontinuation according to the dichotomous OLST groups ( 0 to $<60$ vs. $\geq 60 \mathrm{~s})$ were calculated. A $p$ value $<0.05$ (two-sided) was considered to indicate statistical significance. All statistical analyses were performed using SAS version 9.4 (SAS Institute Inc., Cary, NC, USA). 


\section{RESULTS}

\section{Clinical Characteristics}

The clinical characteristics of the 43 (30 male; 13 female) patients are shown in Table 1 . For all patients, the mean (SD) age and duration of diabetes were 54 (13) years and 11.6 (9.7) years, respectively. On average, patients in the Discontinued group $(n=29)$ had a shorter duration of diabetes, lower fat mass and higher skeletal muscle mass than those in the Continued group $(n=14)$. Abnormal ankle reflexes and retinopathy were less frequent in the Discontinued group than in the Continued group. Other clinical variables, including participation in supervised exercise programs, fasting serum C-peptide levels, eGFR, serum lipid level and albuminuria did not differ between the Continued group and the Discontinued group.

\section{Physical Ability}

Patients in the Discontinued group had better values for the physical ability parameters, including leg muscle quality, OLST, whole-body reaction time and maximal oxygen uptake than did those in the Continued group (Table 2).

\section{Glycemic Status and Antihyperglycemic Medication}

At admission, HbA1c and fasting and mean PG did not differ between the Continued group and the Discontinued group (Table 2). Although fewer patients in the Continued group than in the Discontinued group received insulins (71 vs. $100 \% ; p=0.008)$, total daily doses of insulins were higher in the Continued group than in the Discontinued group at admission $(14 \pm 9$ vs. $22 \pm 15$ units/day; $p=0.022$ ). All five patients who were treated with GLP-1RAs at admission were in the Continued group. Treatment with oral glucose-lowering agents did not differ between the two groups at admission (Table 2).

At discharge, mean PG levels were significantly higher in patients in the Continued group than in those in the Discontinued group (Table 2), although GLP-1RA treatment was initiated in two additional patients in the Continued group and pioglitazone use was more frequent in the Continued group than in the Discontinued group (36 vs. $7 \% ; p=0.028$ ). Total daily doses of insulins in the Continued group remained unchanged at discharge (Table 3).

\section{The Relation Between Physical Ability and Discontinuation of Injectable Medications}

In a stepwise logistic regression analysis, significantly different variables between the Continued group and the Discontinued group (i.e., duration of diabetes, fat mass, skeletal muscle mass, abnormal ankle reflex, retinopathy, leg muscle quality, OLST, whole-body reaction time, maximal oxygen uptake and total daily doses of insulins) were included as potential candidates of explanatory variables. A total of 31 patients for whom data on all of the explanatory variables were available were included in this analysis. The results showed that only the OLST was significantly associated with the discontinuation of injectable medication $(0$ to $<60$ vs. $\geq 60 \mathrm{~s}$; OR $18.9 ; 95 \%$ CI 2.0-178.8; $p=0.011)$.

\section{DISCUSSION}

In the study reported here we examined patients with inadequately controlled T2D across a wide age range who were being treated with injectable medications, such as insulins and GLP-1RAs, at admission. We found that the physical ability parameters of those patients who discontinued injectable medications during their hospital stay were better than those of patients who continued injectable medications during their hospital stay. A stepwise logistic regression analysis that included all of the variables that were significantly different between the Continued group and the Discontinued group revealed that of the physical ability parameters analyzed, only the OLST was a significant variable for injectable medication discontinuation. HbA1c and fasting and mean PG levels did not differ between the Continued 
Table 1 Clinical characteristics of all patients and of patients who discontinued and continued injectable medications, respectively, during the hospital stay

\begin{tabular}{|c|c|c|c|c|}
\hline $\begin{array}{l}\text { Clinical characteristics of } \\
\text { patients }\end{array}$ & All patients $(n=43)$ & $\begin{array}{l}\text { Discontinued group } \\
(n=29)^{\mathrm{a}}\end{array}$ & $\begin{array}{l}\text { Continued group } \\
(n=14)^{\mathrm{a}}\end{array}$ & $p$ value \\
\hline Hospital stay (days) & $15 \pm 5$ & $14 \pm 4$ & $17 \pm 6$ & 0.145 \\
\hline Male gender & $30(69.8 \%)$ & $23(79.3 \%)$ & $7(50.0 \%)$ & 0.077 \\
\hline Age (years) & $54 \pm 13$ & $52 \pm 12$ & $59 \pm 14$ & 0.106 \\
\hline Diabetes duration (years) & $11.6 \pm 9.7$ & $9.2 \pm 8.5$ & $15.9 \pm 10.4$ & $0.038^{*}$ \\
\hline $\begin{array}{l}\text { Alcohol use (never/former/ } \\
\text { current) }\end{array}$ & $\begin{array}{l}22 / 4 / 17(51.2 \% / 9.3 \% / \\
39.5 \%)\end{array}$ & $\begin{array}{l}13 / 4 / 12(44.8 \% / 13.8 \% / \\
\quad 41.4 \%)\end{array}$ & $\begin{array}{l}\text { 9/0/5 (64.3\%/0.0\%/ } \\
\quad 35.7 \%)\end{array}$ & 0.354 \\
\hline $\begin{array}{l}\text { Smoking history (never/former/ } \\
\text { current) }\end{array}$ & $\begin{array}{l}17 / 14 / 12(39.5 \% / 32.6 \% / \\
27.9 \%)\end{array}$ & $\begin{array}{l}11 / 10 / 8(37.9 \% / 34.5 \% / \\
27.6 \%)\end{array}$ & $\begin{array}{l}\text { 6/4/4 (42.9\%/28.6\%/ } \\
28.6 \%)\end{array}$ & 1.000 \\
\hline \multicolumn{5}{|l|}{ Supervised exercise programs } \\
\hline Stretching/balance/resistance & $8(18.6 \%)$ & $6(20.7 \%)$ & $2(14.3 \%)$ & 1.000 \\
\hline $\begin{array}{l}\text { Stretching/balance/resistance/ } \\
\text { aerobic }\end{array}$ & $35(81.4 \%)$ & $23(79.3 \%)$ & $12(85.7 \%)$ & 1.000 \\
\hline Body mass index $\left(\mathrm{kg} / \mathrm{m}^{2}\right)$ & $25.6 \pm 4.5$ & $25.0 \pm 3.8$ & $26.5 \pm 5.7$ & 0.362 \\
\hline Fat mass (\%) & $29.7 \pm 8.2$ & $27.8 \pm 7.4$ & $33.7 \pm 8.8$ & $0.025^{*}$ \\
\hline Skeletal muscle mass (\%) & $38.3 \pm 4.8$ & $39.7 \pm 4.4$ & $35.5 \pm 4.5$ & $0.006^{*}$ \\
\hline $\begin{array}{l}\text { Fasting serum C-peptide } \\
\qquad(\mathrm{nmol} / \mathrm{l})\end{array}$ & $0.5 \pm 0.3$ & $0.5 \pm 0.2$ & $0.6 \pm 0.4$ & 0.590 \\
\hline eGFR $\left(\mathrm{mi} / \mathrm{min} / 1.73 \mathrm{~m}^{2}\right)$ & $78.8 \pm 21.0$ & $78.6 \pm 18.8$ & $79.2 \pm 25.8$ & 0.930 \\
\hline Total cholesterol $(\mathrm{mmol} / \mathrm{l})$ & $4.7 \pm 1.0$ & $4.7 \pm 1.0$ & $4.6 \pm 1.2$ & 0.781 \\
\hline Triglycerides (mmol/l) & $1.7 \pm 0.9$ & $1.7 \pm 1.0$ & $1.7 \pm 0.8$ & 0.858 \\
\hline HDL-cholesterol (mmol/l) & $1.2 \pm 0.4$ & $1.2 \pm 0.4$ & $1.2 \pm 0.4$ & 0.761 \\
\hline Sensory symptoms & $12(27.9 \%)$ & $6(20.7 \%)$ & $6(42.9 \%)$ & 0.160 \\
\hline Abnormal ankle reflex & $22(51.2 \%)$ & $10(34.5 \%)$ & $12(85.7 \%)$ & $0.003^{*}$ \\
\hline Reduced touch perception & $15(34.9 \%)$ & $10(34.5 \%)$ & $5(35.7 \%)$ & 1.000 \\
\hline Retinopathy & $12(27.91 \%)$ & $5(17.2 \%)$ & 7 (50.0\%) & $0.035^{*}$ \\
\hline
\end{tabular}


Table 1 continued

\begin{tabular}{lclll}
\hline $\begin{array}{l}\text { Clinical characteristics of } \\
\text { patients }\end{array}$ & All patients $(\boldsymbol{n}=\mathbf{4 3})$ & $\begin{array}{l}\text { Discontinued group } \\
(\boldsymbol{n}=\mathbf{2 9})^{\mathbf{a}}\end{array}$ & $\begin{array}{l}\text { Continued group } \\
(\boldsymbol{n}=\mathbf{1 4})^{\mathbf{a}}\end{array}$ & $\boldsymbol{p}$ value \\
\hline $\begin{array}{l}\text { Albuminuria (normo/ } \\
\text { micro/macro) }\end{array}$ & $31 / 6 / 6(72.1 \% / 14.0 \% /$ & $23 / 3 / 3(79.3 \% / 10.3 \% /$ & $8 / 3 / 3(57.1 \% / 21.4 \% /$ & 0.323 \\
& $14.0 \%)$ & $10.3 \%)$ & $21.4 \%)$ & \\
\hline
\end{tabular}

Data are presented as a number with the percentage in parenthesis or as the mean \pm standard deviation (SD)

eGFR Estimated glomerular filtration rate, $H D L$ high-density lipoprotein

*Significant difference between Continued group and Discontinued group at $p<0.05$ (two-sided)

a Patients were divided into two groups for comparison: (1) Continued group, which comprised patients who continued receiving injectable medications during hospitalization; Discontinued group, which comprised patients who discontinued treatment with injectable medications during hospitalization

${ }^{b}$ Normoalbuminuria is defined as a urine albumin to creatinine ratio (UACR) of $<30 \mathrm{mg} / \mathrm{g}$ or $<3.4 \mathrm{mg} / \mathrm{mmol}$. Microalbuminuria is defined as a UACR of $30-299 \mathrm{mg} / \mathrm{g}$ or $3.4-33.8 \mathrm{mg} / \mathrm{mmol}$. Macroalbuminuria is defined as a UACR of $\geq 300 \mathrm{mg} / \mathrm{g}$ or $\geq 33.9 \mathrm{~g} / \mathrm{mmol}$

Table 2 Physical ability parameters and glycemic status at admission and at discharge of all patients and of patients who discontinued and continued injectable medications, respectively, during the hospital stay

\begin{tabular}{|c|c|c|c|c|}
\hline $\begin{array}{l}\text { Physical ability parameters and } \\
\text { glycemic status }\end{array}$ & $\begin{array}{l}\text { All patients } \\
(n=43)\end{array}$ & $\begin{array}{l}\text { Discontinued group } \\
(n=29)\end{array}$ & $\begin{array}{l}\text { Continued group } \\
(n=14)\end{array}$ & $p$ value \\
\hline Leg muscle quality ${ }^{\mathrm{a}}$ & $6.3 \pm 1.8$ & $6.8 \pm 1.8$ & $5.4 \pm 1.4$ & $0.023^{*}$ \\
\hline OLST test $(\mathrm{s})(0$ to $<60 / \geq 60)$ & $\begin{array}{l}26 / 17(60.5 \% / \\
39.5 \%)\end{array}$ & $13 / 16(44.8 \% / 55.2 \%)$ & $13 / 1(92.9 \% / 7.1 \%)$ & $0.001^{*}$ \\
\hline Whole-body reaction time (s) & $0.434 \pm 0.174$ & $0.383 \pm 0.084$ & $0.520 \pm 0.246$ & $0.022^{*}$ \\
\hline Maximal oxygen uptake $(\mathrm{ml} / \mathrm{kg} / \mathrm{min})$ & $19.8 \pm 6.1$ & $21.6 \pm 6.1$ & $16.6 \pm 4.9$ & $0.017^{*}$ \\
\hline \multicolumn{5}{|l|}{ HbAlc at admission } \\
\hline HbAlc (\%) & $10.7 \pm 2.3$ & $10.9 \pm 2.3$ & $10.2 \pm 2.1$ & 0.352 \\
\hline $\mathrm{HbAlc}(\mathrm{mmol} / \mathrm{mol})$ & $91.4 \pm 23.9$ & $93.8 \pm 24.6$ & $86.3 \pm 22.3$ & 0.342 \\
\hline \multicolumn{5}{|l|}{ Fasting PG (mmol/l) } \\
\hline At admission & $8.7 \pm 2.8$ & $8.3 \pm 2.7$ & $9.6 \pm 2.8$ & 0.139 \\
\hline At discharge & $6.1 \pm 1.2$ & $6.0 \pm 0.9$ & $6.3 \pm 1.7$ & 0.401 \\
\hline \multicolumn{5}{|l|}{ Mean PG (mmol/l) } \\
\hline At admission (8-point profile) & $11.1 \pm 3.9$ & $10.5 \pm 3.8(1)$ & $12.4 \pm 3.9$ & 0.148 \\
\hline At discharge (7-point profile) & $7.2 \pm 1.5$ & $6.9 \pm 1.1$ & $8.0 \pm 2.0$ & $0.022^{*}$ \\
\hline
\end{tabular}

Data are presented as a number with the percentage in parenthesis or as the mean $\pm \mathrm{SD}$

$H b A c l$ Gycated hemoglobin, OLST one-leg standing time with eyes open, $P G$ plasma glucose

*Significant difference between Continued group and Discontinued group at $p<0.05$ (two-sided)

$a^{a}$ Leg muscle quality is knee extension strength per unit of leg muscle mass 
Table 3 Antihyperglycemic medications at admission and at discharge among all patients and among patients who discontinued and continued injectable medications, respectively, during the hospital stay

\begin{tabular}{|c|c|c|c|c|c|c|}
\hline \multirow{2}{*}{$\begin{array}{l}\text { Antihyperglycemic } \\
\text { medications }\end{array}$} & \multicolumn{3}{|l|}{ At admission } & \multicolumn{3}{|l|}{ At discharge } \\
\hline & $\begin{array}{l}\text { Discontinued } \\
\text { group }(n=29)\end{array}$ & $\begin{array}{l}\text { Continued } \\
\text { group }(n=14)\end{array}$ & $p$ value & $\begin{array}{l}\text { Discontinued } \\
\text { group }(n=29)\end{array}$ & $\begin{array}{l}\text { Continued } \\
\text { group }(n=14)\end{array}$ & $p$ value \\
\hline GLP-1RAs & $0(0 \%)$ & $5(36 \%)$ & $0.002^{*}$ & $0(0 \%)$ & $7(50 \%)$ & $0.002^{*}$ \\
\hline Insulins & $29(100 \%)$ & $10(71 \%)$ & $0.008^{*}$ & $0(0 \%)$ & $8(57 \%)$ & $<0.001^{*}$ \\
\hline $\begin{array}{l}\text { Total daily doses of } \\
\text { insulins (units/day) }\end{array}$ & $14 \pm 9$ & $22 \pm 15$ & $0.022^{*}$ & - & $22 \pm 15$ & - \\
\hline $\begin{array}{l}\text { Total number of insulin } \\
\text { (injections/day) }\end{array}$ & $3 \pm 1$ & $3 \pm 2$ & 0.963 & - & $3 \pm 2$ & - \\
\hline Sulfonylureas & $0(0)$ & $0(0 \%)$ & - & $0(0 \%)$ & $0(0 \%)$ & - \\
\hline Metformin & $12(41 \%)$ & $9(64 \%)$ & 0.203 & $29(100 \%)$ & $14(100 \%)$ & - \\
\hline Pioglitazone & $4(14 \%)$ & $3(21 \%)$ & 0.665 & $2(7 \%)$ & $5(36 \%)$ & $0.028^{*}$ \\
\hline$\alpha$-Glucosidase inhibitors & $1(3 \%)$ & $0(0 \%)$ & 1.000 & $0(0 \%)$ & $0(0 \%)$ & - \\
\hline DPP-4 inhibitors & $6(21 \%)$ & $2(14 \%)$ & 1.000 & $5(17 \%)$ & $2(14 \%)$ & 1.000 \\
\hline SGLT2 inhibitors & $1(3 \%)$ & $0(0 \%)$ & 1.000 & $6(21 \%)$ & $6(43 \%)$ & 0.160 \\
\hline
\end{tabular}

Data are presented as a number with the percentage in parenthesis or as the mean $\pm S D$

$D P P-4$ Dipeptidyl peptidase 4, GLP-1RA glucagon-like peptide-1 receptor agonist, $S G L T 2$ sodium-glucose cotransporter ${ }^{*}$ Significant difference between Continued group and Discontinued group at $p<0.05$ (two-sided)

group and the Discontinued group at admission, but the mean PG levels at discharge were significantly higher in the Continued group than in the Discontinued group. Thus, patients with poor glycemic control and impaired functional balance, as indicated by a short OLST, seemed to bear an increasing burden of pharmacologic medications needed to achieve better glycemic control. In addition, although fasting serum C-peptide levels did not differ between the two groups, total daily doses of insulins were higher in the Continued group than in the Discontinued group at admission and remained unchanged at discharge. Since patients in the Continued group demonstrated lower skeletal muscle mass and higher fat mass than those in the Discontinued group at admission (Table 1), it is possible that patients in the Continued group were more insulin resistant than those in the Discontinued group [16]. However, the association of insulin resistance with impaired functional balance in T2D is beyond the scope of the current study and warrants investigation in future studies.

Evidence suggests that increased physical activity and acute bouts of exercise improve insulin action in muscle and liver [17]. Likewise, reducing sitting time improves glycemic control in patients with T2D [18]. In contrast, a previous study demonstrated that poor mobility with a high fall risk is linked to hyperglycemic status even in younger patients with a short duration of T2D [19]. Also, low cardiorespiratory fitness is associated with an increase in the risk of having metabolic syndrome and T2D, independent of physical activity and sedentary time [20]. Therefore, impaired physical ability in addition to physical inactivity and a sedentary lifestyle may warrant more attention from healthcare providers in terms of glycemic management individualized for each patient with T2D. In this context, OLST is a simple, safe and widely used clinical measure of physical ability (functional balance) that does not require specific devices 
[21]. At our center, OLST is measured approximately 3 months after discharge and annually thereafter in order to identify any significant improvement or deterioration. It would be of interest to compare the longitudinal changes in OLST with the pharmacologic therapy needed to achieve glycemic goals.

We assessed physical ability-related components of physical fitness, including leg muscle strength, functional balance, agility and cardiopulmonary fitness, and obtained estimates of these components by measuring leg muscle quality, OLST, whole-body reaction time and maximal oxygen uptake, respectively. Although these components were all impaired in patients who continued injectable medications compared to those who discontinued injectable medications during hospitalization, a stepwise selection method found that only OLST was a significant variable for injectable medication discontinuation. Although the exact mechanism of this association remains unclear, it is well known that patients with postural instability have gait disturbance [22] and a fear of falling [23]. Therefore, these patients may not be able to reap the full benefits of the supervised exercise program prescribed during hospitalization. Clearly, the assumption that physical ability influences the blood glucose-lowering effect of exercise among patients with T2D should be tested in future studies.

\section{Limitations}

There are a number of limitations to our study. First, the small number of subjects is a major limitation. We applied a fairly high number of exclusion criteria with the aim to exclude patients who were assumed to require insulin therapy under specific conditions at admission, such as metabolic crisis, absolute insulin deficiency, impaired renal function, inflammation and/or liver cirrhosis. Although the use of such strict criteria may have limited the generalizability of the results, they may, in turn, have been reasonably effective at reducing the impact of such potential confounders on the observed association. Second, this was a cross- sectional study and, consequently, causal relationships of the findings could not be determined. Additionally, one may speculate that the current observation is only an incidental relationship among a wide variety of tests, possibly derived from a priori assumptions. However, patients who discontinued injectable medications indeed had performed better on all the physical ability parameters and achieved better glycemic control at discharge than those who continued injectable medications. A stepwise selection method that included all of the variables that were significantly different between the Continued group and the Discontinued group as candidate variables revealed that only OLST was a significant variable for injectable medication discontinuation. Third, diabetic peripheral and autonomic neuropathy can negatively affect functional balance in multiple ways. The results from our previous study demonstrated that a short OLST is associated with peripheral and cardiac autonomic nerve dysfunction and clinical neuropathy in patients with T2D, independent of age [24]. In the current study, abnormal ankle reflexes were more frequent in patients in the Continued group than in those in the Discontinued group. Therefore, the possible association between the presence of diabetic neuropathy and injectable medication continuation may confound the observed results and should also be tested in future studies. Lastly, there may also be a concern for the external validity of the current association. All patients were treated by a diabetologist with general glycemic goals set at preprandial and 2 -h postprandial PG levels of 4.4-7.2 and $<10.0 \mathrm{mmol} /$ 1 , respectively. Their medications were reduced as long as the glycemic goals could be achieved. However, some diabetologists may be reluctant to reduce medications once their patients achieve good glycemic control during a hospital stay. Thus, glycemic treatment could be modified by different pharmacologic approaches used by different diabetologists and be accomplished with more frequent use of injectable medications. 


\section{CONCLUSIONS}

The current study examined insulin- and/or GLP-1RA-treated patients with inadequately controlled T2D who were hospitalized to improve their diabetes self-management ability and who showed preserved endogenous insulin secretion in the absence of clinical abnormalities assumedly requiring insulin therapy across a wide range of ages. Among the physical ability parameters examined, OLST appeared to be associated with injectable medication discontinuation during hospitalization. OLST could possibly be useful as a novel patient factor when physicians consider de-intensification of injectable medication in patients with T2D. Further studies with larger sample sizes are needed to investigate the external validity and potential confounders in this association.

\section{ACKNOWLEDGEMENTS}

The authors gratefully acknowledge the contribution of Izumi Furukawa for data collection and analysis. We would also like to thank the participants of the study.

Funding. No funding or sponsorship was received for this study or the journals rapid service fee.

Authorship. All named authors meet the International Committee of Medical Journal Editors (ICMJE) criteria for authorship for this article, take responsibility for the integrity of the work as a whole, and have given their approval for this version to be published.

Authorship Contributions. Kazuhiro Sugimoto contributed to the study design, data collection, analysis and interpretation of data, and to writing and approving the manuscript. Takashi Sozu and Hiroshi Nishiyama provided statistical consulting and contributed to the writing and approvalof the manuscript. Yuji Tanaka conducted statistical analyses. Takehiko Hoshino performed the physical ability tests. Yuko Watanabe, Akira Tamura, Toshiro
Yamazaki and Setsu Ohta contributed to data collection and final approval of the manuscript. Susumu Suzuki and Takuro Shimbo contributed to reviewing the draft and final approval of the manuscript.

Prior Presentation. This study was in part presented at the 62nd Annual Meeting of the Japan Diabetes Society held in Sendai, Japan, 23-25 May 2019.

Disclosures. Kazuhiro Sugimoto, Yuji Tanaka, Takashi Sozu, Hiroshi Nishiyama, Takehiko Hoshino, Yuko Watanabe, Akira Tamura, Setsu Ohta, Toshiro Yamazaki, Susumu Suzuki and Takuro Shimbo have nothing to disclose.

Compliance with Ethics Guidelines. All analyses were conducted with the approval of the Ethical Committee of the Ohta Nishinouchi Hospital (approval no; 3, approval date; 2018-06-18) and in accordance with Helsinki Declaration of 1964 and its later amendments as well as with the ethical guidelines for medical and health research involving human subjects issued by the Ministry of Health, Labour and Welfare of Japan in 2017.

Data Availability. The datasets used and/or analyzed during the current study are available from the corresponding author on reasonable request.

Open Access. This article is licensed under a Creative Commons Attribution-NonCommercial 4.0 International License, which permits any non-commercial use, sharing, adaptation, distribution and reproduction in any medium or format, as long as you give appropriate credit to the original author(s) and the source, provide a link to the Creative Commons licence, and indicate if changes were made. The images or other third party material in this article are included in the article's Creative Commons licence, unless indicated otherwise in a credit line to the material. If material is not included in the article's Creative Commons licence and your intended use is not permitted by statutory regulation or exceeds the permitted use, you 
will need to obtain permission directly from the copyright holder. To view a copy of this licence, visit http://creativecommons.org/licenses/by$\mathrm{nc} / 4.0 /$.

\section{REFERENCES}

1. Zanuso S, Jimenez A, Pugliese G, Corigliano G, Balducci S. Exercise for the management of type 2 diabetes: a review of the evidence. Acta Diabetol. 2010;47(1):15-22.

2. Colberg SR, Sigal RJ, Yardley JE, et al. Physical activity/exercise and diabetes: a position statement of the American Diabetes Association. Diabetes Care. 2016;39(11):2065-79.

3. Thornton JS, Fremont P, Khan K, et al. Physical activity prescription: a critical opportunity to address a modifiable risk factor for the prevention and management of chronic disease: a position statement by the Canadian Academy of Sport and Exercise Medicine. Br J Sports Med. 2016;50(18): 1109-14.

4. Orlando G, Balducci S, Bazzucchi I, Pugliese G, Sacchetti M. Neuromuscular dysfunction in type 2 diabetes: underlying mechanisms and effect of resistance training. Diabetes Metab Res Rev. 2016;32(1):40-50.

5. Yeung $\mathrm{CHC}$, Au Yeung SL, Fong SSM, Schooling CM. Lean mass, grip strength and risk of type 2 diabetes: a bi-directional mendelian randomisation study. Diabetologia. 2019;62(5):789-99.

6. Kalyani RR, Tian J, Xue QL, et al. Hyperglycemia and incidence of frailty and lower extremity mobility limitations in older women. J Am Geriatr Soc. 2012;60(9):1701-7.

7. Fujinuma $H$, Abe $R$, Yamazaki $T$, et al. Effect of exercise training on doses of oral agents and insulin. Diabetes Care. 1999;22(10):1754-5.

8. Levin PA, Nguyen H, Wittbrodt ET, Kim SC. Glucagon-like peptide-1 receptor agonists: a systematic review of comparative effectiveness research. Diabetes Metab Syndr Obes. 2017;10:123-39.

9. Abd El Aziz MS, Kahle M, Meier JJ, Nauck MA. A meta-analysis comparing clinical effects of short- or long-acting GLP-1 receptor agonists versus insulin treatment from head-to-head studies in type 2 diabetic patients. Diabetes Obes Metab. 2017;19(2): 216-27.
10. American Diabetes Association. 9. Pharmacologic approaches to glycemic treatment: standards of medical care in diabetes-2020. Diabetes Care. 2020;43(Suppl 1):S98-110.

11. Leighton E, Sainsbury CA, Jones GC. A practical review of C-peptide testing in diabetes. Diabetes Ther. 2017;8(3):475-87.

12. Marra M, Sammarco R, De Lorenzo A, et al. Assessment of body composition in health and disease using bioelectrical impedance analysis (BIA) and dual energy X-ray absorptiometry (DXA): a critical overview. Contrast Media Mol Imaging. 2019;2019: 3548284.

13. Becroft L, Ooi G, Forsyth A, King S, Tierney A. Validity of multi-frequency bioelectric impedance methods to measure body composition in obese patients: a systematic review. Int J Obes (Lond). 2019;43(8):1497-507.

14. Park SW, Goodpaster BH, Strotmeyer ES, et al. Decreased muscle strength and quality in older adults with type 2 diabetes: the health, aging, and body composition study. Diabetes. 2006;55(6): 1813-8.

15. Miyatake N, Sakano N, Yoshioka A, Hirao T, Numata T. Evaluation of whole body reaction time and one leg with eye closed balance in elderly Japanese. Open J Epidemiol. 2012;2(01):22.

16. Kim K, Park SM. Association of muscle mass and fat mass with insulin resistance and the prevalence of metabolic syndrome in Korean adults: a cross-sectional study. Sci Rep. 2018;8(1):2703.

17. Roberts CK, Hevener AL, Barnard RJ. Metabolic syndrome and insulin resistance: underlying causes and modification by exercise training. Compr Physiol. 2013;3(1):1-58.

18. Duvivier BM, Schaper NC, Hesselink MK, et al. Breaking sitting with light activities vs structured exercise: a randomised crossover study demonstrating benefits for glycaemic control and insulin sensitivity in type 2 diabetes. Diabetologia. 2017;60(3):490-8.

19. Oliveira PP, Fachin SM, Tozatti J, Ferreira MC, Marinheiro LP. Comparative analysis of risk for falls in patients with and without type 2 diabetes mellitus. Rev Assoc Med Bras (1992). 2012;58(2):234-9.

20. van der Velde J, Schaper NC, Stehouwer CDA, et al. Which is more important for cardiometabolic health: sedentary time, higher intensity physical activity or cardiorespiratory fitness? The Maastricht Study. Diabetologia. 2018;61(12):2561-9. 
21. Michikawa T, Nishiwaki Y, Takebayashi T, Toyama Y. One-leg standing test for elderly populations. J Orthop Sci. 2009;14(5):675-85.

22. Allet L, Armand S, de Bie RA, Golay A, Monnin D, Aminian $\mathrm{K}$, et al. The gait and balance of patients with diabetes can be improved: a randomised controlled trial. Diabetologia. 2010;53(3):458-66.
23. Allet L, Armand S, de Bie RA, et al. Gait alterations of diabetic patients while walking on different surfaces. Gait Posture. 2009;29(3):488-93.

24. Sugimoto K, Hoshino T, Tamura A, Yamazaki T, Suzuki S, Shimbo T. The relationship of one-leg standing time with peripheral nerve function and clinical neuropathy in patients with type 2 diabetes. Diabetol Int. 2018;9(4):243-56. 